\title{
FINITE ELEMENT ERROR ESTIMATES FOR NONLINEAR ELLIPTIC PROBLEMS OF MONOTONE TYPE
}

\author{
Shue-Sum ChOW
}

In this thesis, we examine the order of convergence of finite element approximations to some nonlinear elliptic problems of monotone type. This is determined by establishing error estimates in the 'energy' norm for the approximations. The nonlinear problems we are interested in arise in many practical situations. Examples from blast furnace gas flow, magnetostatic distribution and nonlinear seepage flow are discussed in some details.

In Chapter 2 we show that a class of second order boundary value problems of divergence form with gradient nonlinearity may be formulated variationally, and, in an abstract setting, as an operator equation, if the nonlinear coefficients of the equation satisfy certain conditions. Under these conditions the operator turns out to be monotone. In Chapter 3 we introduce the concept of strong monotonicity and $T$-continuity and demonstrate the relation between strong monotonicity and convexity. We then prove the strong monotonicity and Hölder continuity of the operator introduced in Chapter 2. The well-posedness of the boundary value problem is then shown by establishing the unique solvability and stability of the problem.

In Chapter 4, after showing how error estimates for finite element approximations may be obtained in an abstract setting if the associated operator is strongly monotone and $\mathrm{T}$-continuous, we proceed to derive error

Received 13 October 1983. Thesis submitted to Australian National University, April 1983. Degree approved, September 1983. Supervisors: Dr R.S. Anderssen and Dr F.R. de Hoog.

Copy right Clearance Centre, Inc. Serial-fee code: 0004-9727/83 $\$$ A2. $00+0.00$. 
estimates for the class of nonlinear problems described earlier and examine the order of convergence of the finite element approximations under the assumption that the (weak) solution satisfies only a weak regularity condition.

As the error estimates derived in Chapter 4 are optimal (in the sense that the orders of convergence correspond with those for nondegenerate Linear equations) only for problems whose underlying solution space is a Hilbert space, it is of interest to consider the optimality of the order of convergence in other cases. In Chapter 5 we establish the conditions under which a class of nondegenerate equations, including Ergun's equation and nonlinear seepage flow equations, can be shown to have the optimal order of convergence. We also examine the problems of error estimation in $W^{l, 2}$-norm and for a class of problem solvable in the $W^{l, p}$-Sobolev space with $p>2$.

In Chapter 6, we use the method developed earlier to establish a variational formulation for a class of nonlinear vectorial boundary value problems and to derive error estimates for the finite element approximations.

In the last chapter we discuss a direct method for solving a class of one dimensional problem and present some results from numerical experimentation.

Department of Aerospace Engineering and Engineering Mechanics, University of Texas at Austin, Austin, Texas 78712-1085, USA. 